\title{
La violencia contra las mujeres en el marco del terrorismo de Estado en Argentina ${ }^{1}$
}

Violence against women in the framework of State terrorism in Argentina

Violência contra as mulheres no contexto do terrorismo de Estado na Argentina

Violence contre les femmes dans le contexte du terrorisme d'État en Argentine

\begin{tabular}{l|l} 
María Cecilia Rita Villegas & 2 \\
& $\begin{array}{l}\text { Universidad Nacional } \\
\text { de La Plata }\end{array}$
\end{tabular}

Revista Derechos en Acción

Año 3/Nº 9 Primavera 2018, 251-265

DOl: https://doi.org/10.24215/25251678e220

ORCID: https://orcid.org/0000-0002-8740-4723

Recibido: 29/09/2018

Recibido con modificaciones: 15/10/2018

Aprobado: 15/11/2018

Resumen: Un 30 por ciento de las víctimas del terrorismo de Estado ejercido por la dictadura cívico militar instaurada en Argentina a partir del 24 de marzo de 1976 fueron mujeres, según los datos recabados por la CONADEP (CONADEP, 1991, p. 294). La violencia sistemática sufrida por las mujeres en los centros clandestinos de detención de aquellos años fue específica, sistemática y planificada, tuvo claras intenciones, partió de estereotipos de género, y los profundizó en nuestra sociedad. Los delitos contra la integridad sexual de las víctimas cometidos por los

1 Seminario: Violencia contra las Mujeres y las Niñas. Diplomado en Género y Justicia Profesora Coordinadora: Susana Chiarotti. Año 2018.

2 Abogada egresada de la Facultad de Ciencias Jurídicas y Sociales de la UNLP, integrante del Ministerio Público de la Nación. 
represores no fueron considerados partes del plan sistemático contra la población, en el marco de la persecución política e ideológica desatada en esos años, si no como hechos aislados. Recién en 2010, la violencia sexual, en este contexto, fue tratada como delito de lesa humanidad. Los objetivos de mi propuesta serán identificar los distintos estereotipos de género presentes en el referido accionar del Estado, determinar si dichos estereotipos siguen vigentes en la actualidad, analizar las causas por las cuáles la violencia contra las mujeres durante el terrorismo de Estado quedó invisibilizada en el sistema judicial argentino, y por último examinar los avances del Estado Argentino en la materia con posterioridad a las recomendaciones efectuadas en 2010 por el Comité de Derechos Humanos y el Comité de la CEDAW.

Palabras claves: Terrorismo de Estado; violencia contra mujeres; estereotipos de género.

Abstract: According to data collected by CONADEP (CONADEP, 1991, p. 294), 30 percent of the victims of state terrorism by the civic-military dictatorship established in Argentina on March 24, 1976, were women. The systematic violence suffered by women in the clandestine detention centers of those years was specific, systematic and planned, had clear intentions, was based on gender stereotypes, and deepened them in our society. The crimes against the sexual integrity of the victims committed by the repressors were not considered part of the systematic plan against the population, within the framework of the political and ideological persecution unleashed in those years, but rather as isolated events. It was not until 2010 that sexual violence, in this context, was treated as a crime against humanity. The objectives of my proposal will be to identify the different gender stereotypes present in the State's actions in question, to determine whether those stereotypes are still valid today, to analyse the reasons why violence against women during State terrorism became invisible in the Argentine judicial system, and finally to examine the progress made by the Argentine State in this area following the recommendations made in 2010 by the Human Rights Committee and the CEDAW Committee

Keywords: State terrorism; violence against women; gender stereotypes.

Resumo: 30 por cento das vítimas do terrorismo de Estado exercido pela ditadura cívico militar na Argentina a partir de 24 de março, 1976 
eram mulheres, de acordo com dados coletados pela Comissão em Disappeared (CONADEP, 1991, p. 294). A violência sistemática sofrida pelas mulheres nos centros de detenção clandestinos daqueles anos foi específica, sistemática e planejada, tinha intenções claras, partiu de estereótipos de gênero e aprofundou-os em nossa sociedade. Crimes contra a integridade sexual das vítimas cometidos pelos repressores não foram considerados parte de um plano sistemático contra a população no contexto da perseguição política e ideológica desencadeada naqueles anos, se os eventos não como isoladas. Somente em 2010, a violência sexual, nesse contexto, foi tratada como um crime contra a humanidade. Os objectivos da minha proposta vai identificar os diferentes estereótipos de gênero presentes na referida ação do Estado, se tais estereótipos ainda são válidas hoje, analisar as razões pelas quais a violência contra as mulheres durante o terrorismo de Estado permaneceu invisível no Sistema judicial argentino e, por fim, examinar o progresso do Estado argentino na matéria, após as recomendações feitas em 2010 pela Comissão de Direitos Humanos e pelo Comitê da CEDAW

Palavras Chaves: Terrorismo Estatal; violência contra as mulheres; estereótipos de gênero

Résumé: 30 pour cent des victimes du terrorisme d'État exercé par la dictature civilo-militaire établie en Argentine le 24 mars 1976 étaient des femmes, selon les données recueillies par la CONADEP (CONADEP, 1991, p.294). La violence systématique subie par les femmes dans les centres de détention clandestins de ces années était spécifique, systématique et planifiée, avait des intentions claires, partait de stéréotypes sexistes et les avait approfondis dans notre société. Les crimes contre l'intégrité sexuelle des victimes commis par les oppresseurs n'étaient pas considérés comme faisant partie du plan systématique contre la population, dans le cadre de la persécution politique et idéologique déclenchée au cours de ces années, sinon comme des incidents isolés. Ce n'est qu'en 2010 que la violence sexuelle, dans ce contexte, a été traitée comme un crime contre l'humanité. Les objectifs de ce travail seront d'identifier les différents stéréotypes de genre présents dans les actions susmentionnées de l'État, de déterminer si ces stéréotypes sont toujours valables aujourd'hui et d'analyser les raisons pour lesquelles la violence à l'égard des femmes pendant le terrorisme d'État a été dissimulée dans le système judiciaire argentin. Enfin, examinez les progrès de l'État argentin 
en la matière conformément aux recommandations formulées en 2010 par le Comité des droits de l'homme et le Comité de la CEDAW.

Mot-clés: Terrorisme d'État; violence contre les femmes; stéréotypes de genre.

\section{Introducción}

Un 30 por ciento de las víctimas del terrorismo de Estado ejercido por la dictadura cívico militar instaurada en Argentina a partir del 24 de marzo de 1976 fueron mujeres, según los datos recabados por la CONADEP (CONADEP, 1991, p. 294). La violencia sistemática sufrida por las mujeres en los centros clandestinos de detención de aquellos años fue específica, sistemática y planificada, tuvo claras intenciones, partió de estereotipos de género, y los profundizó en nuestra sociedad. Los delitos contra la integridad sexual de las víctimas cometidos por los represores no fueron considerados partes del plan sistemático contra la población, en el marco de la persecución política e ideológica desatada en esos años, si no como hechos aislados. Recién en 2010, la violencia sexual, en este contexto, fue tratada como delito de lesa humanidad.

Los objetivos de mi propuesta serán identificar los distintos estereotipos de género presentes en el referido accionar del Estado, determinar si dichos estereotipos siguen vigentes en la actualidad, analizar las causas por las cuáles la violencia contra las mujeres durante el terrorismo de Estado quedó invisibilizada en el sistema judicial argentino, y por último examinar los avances del Estado Argentino en la materia con posterioridad a las recomendaciones efectuadas en 2010 por el Comité de Derechos Humanos y el Comité de la CEDAW.

\section{Gobiernos militares y terrorismo de Estado}

Durante el siglo XX, en la República Argentina se impusieron seis golpes de Estado - 1930, 1943, 1955, 1962, 1966 y 1976-. 
Siguiendo a Duhalde (2013, p. 18) destaco que los golpes militares anteriores al de 1976 dieron lugar a gobiernos militares clásicos, de carácter transitorio. El régimen militar instaurado a partir de 1976 se basó en una elaborada teoría que configuró el Estado Terrorista y su faz clandestina permanente. No se trataba ya sólo del Estado militarmente ocupado, donde se establecía un manejo discrecional del aparato del Estado, abrogando derechos y libertades civiles, sino de un profundo cambio en la concepción del Estado, un verdadero nuevo Estado de excepción. El terrorismo de Estado se basó en el crimen sistemático - el terrorcomo forma de disciplinamiento del conjunto social.

\section{Estereotipos de género en el discurso del terrorismo de Estado}

Partiré de la definición de estereotipo, para luego analizar los vigentes en el discurso del terrorismo de Estado.

"Un estereotipo es una visión generalizada o una preconcepción sobre los atributos o características de los miembros de un grupo en particular o sobre los roles que tales miembros deben cumplir" (Cook y Cusack, 2010, p. 11).

Los estereotipos de género actúan reproduciendo la asimetría estructural existente entre hombres y mujeres, que tiene su origen en el sistema patriarcal imperante en nuestro país, que parte de una tajante diferencia entre el espacio público y el privado, y en la adjudicación de un rol secundario para la mujer, relegada siempre a este último y, por ende, subordinada al hombre. Desde esta jerarquización asimétrica de los roles, se desprenden las distintas formas de subordinación y discriminación, y como lógica consecuencia la violencia contra las mujeres.

El terrorismo de Estado se propuso garantizar un capitalismo patriarcal y para ello agudizó los estereotipos ya existentes en nuestra sociedad. Ante el incipiente nuevo modelo femenino que surgía tímidamente en nuestro país en las décadas del 60 y 70, postulando el trabajo remunerado como realización 
personal de la mujer, la aceptación de la sexualidad femenina pre matrimonial y la desacreditación de la condición de ama de casa, el terrorismo de Estado propuso un regreso a los valores tradicionales. Para ello profundizó el rol de la mujer de ama de casa, madre y esposa, remarcándose su función reproductiva, doméstica y de cuidado. El discurso del terrorismo de Estado exacerbó el modelo de mujer garante de la unidad familiar, célula básica de la sociedad. El único ámbito propio para la mujer era el privado. Asimismo ahondó en la dicotomía virgen o prostituta, resignificándola en la dicotomía modelo mariano -virgen María/virgen de Luján- o mujer subversiva -militante política y social. (D`Atri, 2011).

Aucia (2011) encuentra una correlación establecida por el patriarcado entre mala madre, madre abandónica y militante política y social. Describe que para el terrorismo de Estado la mujer víctima de represión era una mujer doblemente transgresora, -de los valores sociales y políticos tradicionalmente constituidos, y del ámbito de lo privado /doméstico, avanzando sobre el espacio público/político, hasta entonces reservado a los hombres.

Delmas (2016) señala que en ese período la representación que se hizo de las mujeres militantes, sindicales, políticas, de organizaciones armadas, fue la de libres y sexualmente activas, malas madres, malas esposas y malas amas de casa. Por ello uno de los insultos más utilizados fue el de "putas". La otra representación, conservadora y represiva sostenida desde la moral católica, fue la de mujeres heteronormativas, monogámicas, reducidas a la vida privada y a funciones de cuidado y de reproducción, siempre sometida a la autoridad masculina.

\section{La violencia contra las mujeres}

Como ya adelanté la violencia sistemática sufrida por las mujeres en los centros clandestinos de detención fue específica, sistemática y planificada. Los métodos represivos fueron distintos para las mujeres, además de las torturas sufrieron violencia 
sexual al decir de Aucia (2011) con carácter rutinario y extendido, con el objetivo principal de disciplinarlas en sus identidades de género. La finalidad fue someter al poder patriarcal manifestado en un orden sexual y político, imprimiendo identidades femeninas en esos cuerpos y castigarlos por haberse apartado de los roles asignados por las relaciones binarias de género. Diferente fue el caso de las agresiones sexuales hacia hombres, en las que el objetivo fue degradarlos y disminuir su status de par en tanto varón, dejándolos en un lugar feminizado y subordinado, como lo es la posesión sexual por un hombre, en la perspectiva patriarcal.

Segato (2012) marca la contradicción existente en pretender resolver los problemas de la agresión a la mujer en la esfera de lo público, en el mismo Estado que privatiza lo sexual, la familia, y por ende, los problemas de las mujeres. El Estado privatiza la familia, la conyugaliza, la transforma en el terreno de la propiedad, de la autoridad privada. La mujer es entonces, mucho más vulnerable y para contrarrestar esa vulnerabilidad incorpora legislación que ya está legislando contra la vulnerabilidad de la mujer que crece a la par del avance de la racionalidad moderna del Estado. En este esquema los temas de género y de violencia sexual quedan en una oscuridad. Esa confusión se origina en la atmósfera patriarcal en la que vivimos, donde lo patriarcal tiene que ver con la moral y con lo privado. Si bien la violencia sexual es básicamente una forma de agresión a la persona, en nuestra sociedad se impuso como una agresión moral. La confusión entre la dimensión moral y la dimensión puramente bélica de la agresión sexual, impide actuar pensar soluciones para la cuestión de la violencia de género. Ante esto Segato propone como primer paso entender que la agresión sexual es una agresión entre muchas otras, que no destruye moralmente, sino físicamente. Afirmar que la agresión sexual es una forma de tortura como cualquier otra, es una forma de destrucción del cuerpo como cualquier otra, es una destrucción de la autonomía como cualquier otra, y no una destrucción moral de la persona. La construcción actual de la agresión sexual a las mujeres no 
sólo tiene que ver con el eje agresor-agredida, sino también con el eje del agresor y sus pares. Por medio del cuerpo de la mujer agredida sexualmente, se produce una agresión a la moral de los hombres, con quienes esta mujer agredida mantiene una relación de dependencia.

\section{La invisibilización de la violencia contra las mujeres durante el terrorismo de Estado en el sistema judicial argentino}

Históricamente el sistema judicial argentino consideró de poca importancia la investigación de delitos sexuales, lo que demuestra la discriminación por género existente, si consideramos que la mayoría de las víctimas son mujeres (Paolini Pecoraro 2011). Conclusión aplicable también a la investigación de violencia sexual denunciada en causas de lesa humanidad.

El Código Penal Argentino, asimismo, exige para la investigación de estos delitos la denuncia de la víctima, requisito que se convirtió en un verdadero obstáculo en el marco de las causas de lesa humanidad.

En los testimonios brindados ante organismos de derechos humanos, en sede judicial o ante la CONADEP, existieron referencias a la violencia sexual ejercida por el terrorismo de Estado. Sin embargo este accionar quedó subsumido en la figura de tormentos o tortura. Corresponde destacar que en los primeros juicios a los represores pareciera apreciarse la supremacía de una identidad militante por sobre la identidad de género de las víctimas.

Balardini Oberlin y Sobredo (2016) explican que en la década del 80 el objetivo general fue probar la represión ilegal. Este enfoque opacó las vivencias individuales que, como los casos de violencia contra las mujeres, quedaron en un claro segundo plano frente a la dimensión amplia del plan sistemático de desaparición y exterminio. Las autoras también reseñan que la falta de sensibilización de los operadores judiciales respecto a estos temas fue otro obstáculo para la judicialización. 
En general al prestar declaración testimonial no se les preguntó a las víctimas si existió violencia sexual, cosa que si se hizo respecto de otros delitos (robos, torturas, ingresos violentos a sus domicilios, etc). En los procesos penales en que las víctimas declararon haber padecido agresiones sexuales, estas denuncias fueron efectuadas de manera espontánea por quienes las sufrieron. Otro obstáculo fueron los niveles de autoría. Estos delitos fueron de los denominados de mano propia y la doctrina mayoritaria le adjudicó por ello la imposibilidad de otras formas de autoría (mediata y coautoría, ya sea paralela o por reparto funcional de tareas).

\section{Las recomendaciones de los Comités de Derechos Humanos y de la CEDAW de 2010 y el avance de la visibilización de los delitos sexuales durante la dictadura}

En el año 2010, dos Comités de Naciones Unidas -de Derechos Humanos y de la CEDAW- emitieron recomendaciones que obligaban al Estado a incorporar la perspectiva de género en el juzgamiento de las violaciones a los derechos humanos cometidos durante la dictadura.

Hasta el año 2010 la violencia sexual desplegada por el terrorismo de Estado quedó reducida a la categoría de delito común y por ello, prescriptible. Como señala Chiarotti (2012) en abril de ese año, por primera vez se incluyó la violencia sexual como parte de un plan sistemático de tortura contra las mujeres víctimas, cuando el Tribunal Oral Federal de Santa Fe condenó al ex agente de inteligencia del Ejército Argentino, Horacio Barcos, a 15 años de prisión por secuestro, privación ilegal de la libertad y torturas. No se reconoció la violencia sexual como delito autónomo. de las torturas. Ese mismo año en la ciudad de Mar del Plata se juzgó a Gregorio Rafael Molina, ex suboficial de la Fuerza Aérea. Molina fue condenado a prisión perpetua por, entre otros delitos, cinco violaciones agravadas y una tentativa. Las víctimas eran dos detenidas. Molina fue autor directo de las violaciones. En este caso, la violencia sexual fue planteada 
de forma autónoma al resto de los tormentos. En Tucumán, también en ese año se dicta procesamiento en la Causa Arsenales Miguel de Azcuénaga, en la que se investigan abusos sexuales y violaciones. En esta causa se procesa por responsabilidad mediata en base a la teoría del dominio del hecho, aclarándose que la no individualización del o los autor/es material/es de los delitos sexuales, no impediría analizar la existencia de otras formas de participación criminal. También en Tucumán, en el año 2011, en la causa Penal de Villa Urquiza, se declaró que las mujeres alojadas en ese Penal durante la vigencia del terrorismo de Estado habrían sido víctimas de actos que califican como las más graves y reprochables formas de violencia contra la mujer (art. 1 y 2 de la Convención de Belém do Pará), ordenando al Ministerio Público Fiscal su investigación. En esa causa se habló de autoría mediata de varios delitos, entre ellos violación sexual agravada en grado reiterado, calificando los delitos en el delito internacional de genocidio, al tenor de la normativa internacional vigente al momento de los hechos. También en 2011 la Cámara Federal de Apelaciones de Mendoza, en los Autos Menéndez y otros, falló que en el caso argentino se dan todos los requisitos necesarios como para considerar a los ataques sexuales contra las mujeres detenidas como delitos de lesa humanidad. Estos delitos fueron imputados en base a la teoría de la autoría mediata a través de aparatos organizados de poder.

Por resolución PGN No 557/12 la Procuración General de la Nación emitió un instructivo a los y las fiscales del país, elaborado por para que tengan en consideración el documento elaborado por la Unidad Fiscal de Coordinación y Seguimiento de las causas por violaciones a los derechos humanos cometidos durante el terrorismo de Estado, Consideraciones sobre el juzgamiento de los abusos sexuales cometidos en el marco del terrorismo de Estado. En este se concluye que los abusos sexuales cometidos como parte de un ataque generalizado o sistemático contra la población civil son crímenes contra la humanidad, sin depender de la frecuencia, sistematicidad o generalidad con que hayan ocurrido. También señala que estos hechos deben 
calificarse de acuerdo a las figuras que el Código Penal prevé especificamente. no resultando correcto subsumirlos como tormentos. Así mismo se analizan cuestiones relativas a la autoría y participación, concluyendo que los abusos sexuales no pueden catalogarse como delitos de propia mano ya que admiten las mismas formas de autoría y participación que otros delitos como por ejemplo el homicidio y los tormentos. En cuanto al requisito de instancia privada, se considera que en aquellos casos en que las víctimas no sobrevivieron, debe tenerse por configurada una de las excepciones previstas a dicho requisitos.

Asimismo por resolución PGN No 3655/15 la Procuración General de la Nación recomendó a los y las fiscales que intervengan en investigaciones sobre violaciones a derechos humanos la observancia de la Guía para los Ministerios Públicos del Mercosur para la interpretación y aplicación de los tratados de asistencia jurídica mutua en asuntos penales relativos a casos de graves violaciones a los derechos bumanos $\mathrm{y}$ la Guía de actuación para los Ministerios Públicos en la investigación penal de casos de violencia sexual perpetrados en el marco de crímenes internacionales, en particular de crimenes de lesa humanidad, adoptadas por la XVII y la XVIII Reunión Especializada de Ministerios Públicos del Mercosur y Estados Asociados. En esta última los Ministerios Públicos acordaron incorporar la perspectiva de género y el enfoque interseccional en la investigación de este tipo de delitos, se estableció la obligación de investigarlos de manera seria, imparcial y efectiva, encuadrándolos en los delitos sexuales específicos, garantizando su visibilidad, e impulsando la persecución penal para imputar a todos los responsables, cualquiera sea su forma de participación y niveles de autoría en la que hayan intervenido. Asimismo se comprometen a promover las medidas necesarias para brindar una reparación integral a las víctimas de violencia sexual conforme es definida por el derecho internacional de los derechos humanos.

Por último en el año 2016 la Dirección General de Políticas de Género y la Dirección General de Derechos Humanos 
del Ministerio Público Fiscal elaboró el documento Aportes del Ministerio Público Fiscal para la actualización del VII Informe Periódico del Estado argentino ante el Comité de la CEDAW. En él se destaca que desde el año 2010 se dictaron 18 sentencias con condenas por crímenes de violencia sexual (17 sentencias definitivas de tribunales orales federales de distintas regiones del país, y una de la Cámara Federal de Casación Penal). Al mes de octubre de 2016 fueron condenadas 78 personas (76 hombres y 2 mujeres) por delitos sexuales y aborto forzado por los casos de 63 víctimas ( 57 mujeres y 6 hombres). En cuanto a la forma de autoría y participación atribuida, solo el 13\% de los condenados lo fue a título de autor directo. Con relación a las medidas de reparación a víctimas de estos delitos se destaca la causa Metán del año 2014, donde se condenó a un imputado como autor mediato y otros dos imputados como autores materiales del delito de violación sexual reiterada contra una niña que había sido sometida a esclavitud sexual y luego fue vendida a un hombre. En la sentencia se hizo lugar a la demanda civil interpuesta por la víctima, y se fijó una indemnización por los rubros de terapia y medicamentos hasta el total restablecimiento de su salud psíquica; daño moral; pérdida de chance laboral; daño psíquico y daño al proyecto de vida. La reparación a las víctimas es la gran deuda pendiente del Estado Argentino.

En el año 2016 los Comités de Derechos Humanos y de la CEDAW emitieron nuevas observaciones, en las que remarcan la existencia de estereotipos de género sobre los roles de las mujeres en la familia. El Comité de Derechos Humanos indicó que el Estado parte debe redoblar sus esfuerzos para eliminar los estereotipos de género sobre el papel y las responsabilidades de los hombres y de las mujeres en la familia y en la sociedad. El Comité CEDAW manifestó que sigue preocupado sobre la persistencia de estereotipos discriminatorios sobre las funciones y responsabilidades de mujeres y hombres en la familia y en la sociedad, las formas de discriminación que se cruzan así como sobre la arraigada cultura de machismo en Argentina, que apuntala la discriminación y la violencia de género contra 
la mujer, como así también las barreras institucionales que enfrentan las mujeres dado los estereotipos discriminatorios, prejuicios judiciales y conocimiento limitado sobre los derechos de la mujer que ostentan la judicatura y la policía.

\section{Conclusiones}

Sin dudas, la falta de investigación y de castigo a los responsables directos e indirectos de la violencia sexual perpetrada contra las mujeres en el marco del terrorismo de Estado facilita y legitima la reproducción de la violencia y de los estereotipos que la generan desde entonces y hasta la actualidad.

Dichos estereotipos estuvieron presentes en los motivos y en la modalidad de la violencia contra las mujeres, como así también en la respuesta del sistema judicial a la misma. Si bien ya existían en la sociedad argentina en los años 70, fueron profundizados por el terrorismo de Estado, persistieron en los juzgamientos de esos delitos y se encuentran presentes en la sociedad actual. Su vigencia garantiza la naturalización de la violencia contra las mujeres y la reproduce. La asimilación de la mujer al ámbito privado, la asunción de los roles domésticos y de cuidado, son patrones que tiene aún una vigencia indiscutible. Los informes del Comité de Derechos Humanos y de la CEDAW de 2016 dan cuenta de la subsistencia de los mismos.

Puedo concluir, de este modo, que existe un vínculo entre la impunidad de la que gozó la violencia contra las mujeres durante el terrorismo de Estado y los niveles de violencia hacia las mujeres actuales. Que recién en el año 2010 se haya podido considerar este tipo de delitos como de lesa humanidad da cuenta de la subsistencia de los mismos estereotipos sexistas en la actualidad. Esta respuesta tardía e insuficiente- es necesario destacar el déficit existente en cuanto a reparación integral para las víctimas de violencia sexual en el contexto referenciado- es en sí misma una discriminación en el acceso a la justicia. El Poder Judicial de este modo, favorece la tolerancia social a la violencia contra las mujeres. 
Sintetizando, la invisibilización de la violencia contra las mujeres en el marco del terrorismo de Estado guarda una estrecha relación con la naturalización de la violencia contra las mujeres.

\section{Bibliografía}

Aucia, A. (2011) Género, violencia sexual y contextos represivos. En Vasallo (Ed.) Grietas en el silencio. En Seminario PRIGEPP Violencia. Recuperado del Programa Regional de Formación en Género y Políticas Públicas (PRIGEPP). http://prigepp.org

Balardini, L, Oberlin, A. y Sobredo L. (2016) Violencia de género y abusos sexuales en centros clandestinos de detención. Un aporte a la comprensión de la experiencia argentina. Recuperado de https://www.cels.org.ar/web/wp-content/ uploads/2016/05/Balardini-Oberlin-Sobredo.pdf

Chiarotti S. (2012) Violencia Sexual en el marco del terrorismo de estado. Recuperado de https://insgenar.files.wordpress. com/2012/12/testimonio-de-contexto-violencia-sexual-en-losccd.pdf

Comité de Derechos Humanos- ONU (2016) Observaciones finales sobre el quinto informe periódico de Argentina. Proyecto preparado por el Comité. Recuperado de http://acnudh.org/wpcontent/uploads/2016/12/CCPR_C_ARG_CO_5_24580_S-2.pdf

Comité para la Eliminación de la Discriminación contra las mujeresONU (2016) Observaciones finales sobre el séptimo informe periódico de Argentina. Recuperado de http://acnudh.org/ wp-content/uploads/2016/12/CEDAW_C_ARG_CO_7_ 25088_E.pdf

CONADEP. (1991) Nunca mas. Informe de la Comisión sobre la desaparición de personas. Buenos Aires, Argentina: EUDEBA.

Cook R. y Cusack S. (2010) Estereotipos de género: Perspectivas Legales Trasnacionales. En Seminario PRIGEPP Violencia. Recuperado del Programa Regional de Formación en Género y Políticas Públicas (PRIGEPP). http://prigepp.org

D’Atri, A. (2011) Las luchas de las mujeres: del genocidio al femicidio. Recuperado de http://andreadatri.blogspot.com.ar 
Delmas, F. (2016) La dictadura en clave de género en. Tram/p]as de la comunicación y la cultura, $\mathrm{N}^{\circ} 78$, marzo 2016. Recuperado de http:www.revistatrampas.com.ar

Dirección General de Políticas de Género y Dirección General de Derechos Humanos del Ministerio Público Fiscal (2016). Aportes del Ministerio Público Fiscal para la actualización del VII Informe Periódico del Estado argentino ante el Comité de la CEDAW. Buenos Aires, Argentina. Recuperado de https:// www.mpf.gob.ar/dgdh/files/2016/11/Aportes-del-MPF-parael-Comit\%C3\%A9-CEDAW.pdf

Duhalde, E. (2013) El Estado terrorista argentino. Buenos Aires, Argentina: Ediciones Colihue.

Paolini Pecoraro A. (2011) Judiciabilidad de los delitos sexuales en el marco de las causas de lesa humanidad. En Vasallo (Ed.) Grietas en el silencio. En Seminario PRIGEPP Violencia. Recuperado del Programa Regional de Formación en Género y Políticas Públicas (PRIGEPP). http://prigepp.org

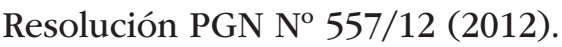

Resolución PGN No 3655/15 (2015).

Segato, R. (2012) La violencia sexual y el discurso del derecho. En Sonderéguer, M. y Correa V. (comp.) Cuaderno de Trabajo Violencia de Género en el terrorismo de Estado: Políticas de Memoria, Justicia y Reparación. Bernal, Argentina: Universidad Nacional de Quilmes. 